\title{
Chemotherapy for pulmonary large cell neuroendocrine carcinomas: does the regimen matter?
}

\author{
Jules L. Derks ${ }^{1}$, Robert Jan van Suylen ${ }^{2}$, Erik Thunnissen ${ }^{3}$ \\ Michael A. den Bakker ${ }^{4,5}$, Harry J. Groen ${ }^{6}$, Egbert F. Smit ${ }^{7,8}$, Ronald \\ A. Damhuis ${ }^{9}$, Esther C. van den Broek ${ }^{10}$, Ernst-Jan M. Speel ${ }^{11,13}$, \\ Anne-Marie C. Dingemans ${ }^{1,13}$ and PALGA group ${ }^{12}$
}

\begin{abstract}
Affiliations: 'Dept of Pulmonary Diseases, GROW School for Oncology and Developmental Biology, Maastricht University Medical Centre, Maastricht, The Netherlands. ${ }^{2}$ Pathology-DNA, Jeroen Bosch Hospital, 's-Hertogenbosch, The Netherlands. ${ }^{3}$ Dept of Pathology, VU University Medical Centre, Amsterdam, The Netherlands. ${ }^{4}$ Dept of Pathology, Maasstad Hospital, Rotterdam, The Netherlands. ${ }^{5}$ Dept of Pathology, Erasmus MC, Rotterdam, The Netherlands. 'Dept of Pulmonary Diseases, University of Groningen and University Medical Centre, Groningen, The Netherlands. ${ }^{7}$ Dept of Pulmonary Diseases, VU Medical Center, Amsterdam, The Netherlands. ${ }^{8}$ Dept of Thoracic Oncology, Netherlands Cancer Institute, Amsterdam, The Netherlands. ${ }^{9}$ Dept of Research, Comprehensive Cancer Association, Utrecht, The Netherlands. ${ }^{10}$ PALGA Foundation, Houten, The Netherlands. ${ }^{11}$ Dept of Pathology, GROW School for Oncology and Developmental Biology, Maastricht University Medical Centre, Maastricht, The Netherlands. ${ }^{12} \mathrm{~A}$ full list of the members of the PALGA group can be found in the Acknowledgements. ${ }^{13}$ Both authors contributed equally.
\end{abstract}

Correspondence: Anne-Marie C. Dingemans, Dept of Pulmonology, P. Debyelaan 25, Postbox 58006202 AZ, Maastricht, The Netherlands. E-mail: a.dingemansamumc.nl

@ERSpublications

Treatment for pulmonary LCNEC is debated; we show favourable results for standard gemcitabine NSCLC chemotherapy http://ow.ly/2Xt730a0PK0

Cite this article as: Derks JL, van Suylen RJ, Thunnissen E, et al. Chemotherapy for pulmonary large cell neuroendocrine carcinomas: does the regimen matter? Eur Respir J 2017; 49: 1601838 [https://doi.org/ $10.1183 / 13993003.01838-2016]$.

ABSTRACT Pulmonary large cell neuroendocrine carcinoma (LCNEC) is rare. Chemotherapy for metastatic LCNEC ranges from small cell lung carcinoma (SCLC) regimens to nonsmall cell lung carcinoma (NSCLC) chemotherapy regimens. We analysed outcomes of chemotherapy treatments for LCNEC.

The Netherlands Cancer Registry and Netherlands Pathology Registry (PALGA) were searched for patients with stage IV chemotherapy-treated LCNEC (2003-2012). For 207 patients, histology slides were available for pathology panel review. First-line platinum-based combined chemotherapy was clustered as "NSCLC-t", comprising gemcitabine, docetaxel, paclitaxel or vinorelbine; "NSCLC-pt", with pemetrexed treatment only; and "SCLC-t", consisting of etoposide chemotherapy.

A panel review diagnosis of LCNEC was established in 128 out of 207 patients. NSCLC-t chemotherapy was administered in 46\% ( $n=60)$, NSCLC-pt in 16\% $(n=20)$ and SCLC-t in 38\% $(n=48)$ of the patients. The median (95\% CI) overall survival for NSCLC-t chemotherapy was 8.5 (7.0-9.9) months, significantly longer than patients treated with NSCLC-pt, with a median survival of 5.9 (5.0-6.9) months (hazard ratio 2.51, 95\% CI 1.39-4.52; $\mathrm{p}=0.002$ ) and patients treated with SCLC-t chemotherapy, with a median survival of 6.7 (5.0-8.5) months (hazard ratio 1.66, 95\% CI 1.08-2.56; $\mathrm{p}=0.020$ ).

In patients with LCNEC, NSCLC-t chemotherapy results in longer overall survival compared to NSCLC-pt and SCLC-t chemotherapy.

This article has supplementary material available from erj.ersjournals.com

Received: Sept 192016 | Accepted after revision: March 052017

Copyright $\odot E R S$ 2017. This ERJ Open article is open access and distributed under the terms of the Creative Commons Attribution Non-Commercial Licence 4.0. 


\section{Introduction}

Pulmonary large cell neuroendocrine carcinoma (LCNEC) is a subtype of lung cancer with neuroendocrine morphology, neuroendocrine differentiation on immunohistochemistry, a high mitotic rate $(>10$ mitosis. $2 \mathrm{~mm}^{-2}$ ) and nonsmall cell cytological features [1]. LCNEC is rare and accounts for $\sim 3 \%$ of all lung cancers, but the proportion of lung cancers diagnosed as LCNEC appears to be increasing [2]. Because the histological features of LCNEC overlap with nonsmall cell lung carcinoma (NSCLC) and occasionally with small cell lung carcinoma (SCLC), histological diagnosis can be difficult $[3,4]$.

Because of the difficulties in diagnosing LCNEC, and its rarity, the optimal systemic treatment has not been adequately established [5]. In the current European Society for Medical Oncology guidelines for NSCLC, no specific treatment for LCNEC is described [6]. In the American Society of Clinical Oncology (ASCO) guideline, either platinum-etoposide chemotherapy treatment (SCLC type) or the same regimen as for nonsmall cell nonsquamous carcinoma (NSCLC type) is advised for LCNEC. However, SCLC-type chemotherapy is considered by expert opinion to be most appropriate [7].

Several observations suggest that LCNEC should respond best to a SCLC-type treatment. For instance, recent studies show that the genomic profile of LCNEC corresponds closely with SCLC $[8,9]$. In addition, we reported that the prognosis and metastatic pattern at diagnosis of LCNEC significantly overlaps with SCLC $[2,10]$. However, important differences in the response to SCLC-type chemotherapy treatment for LCNEC and SCLC have been reported [5]. Two single-arm phase II trials in LCNEC $(\mathrm{n}=29$ and $\mathrm{n}=30)$ showed an objective response rate (ORR) for etoposide or irinotecan combined with cisplatin ranging from $31 \%$ to $47 \%[11,12]$, substantially lower compared to SCLC phase III trials evaluating etoposidecisplatinum chemotherapy (ORR $\approx 66 \%$ ) [13]. Because of the reported higher resistance to SCLC-type chemotherapy in LCNEC, some clinicians favour a NSCLC-type chemotherapy treatment.

Because of these perceived differences, we investigated the chemotherapy treatment of patients with metastatic LCNEC in the Netherlands from 2003 to 2012. Furthermore, we retrospectively correlated the overall survival and progression free survival (PFS) with chemotherapy type in patients with a panel-reviewed histological diagnosis of LCNEC.

\section{Material and methods}

\section{Data sources and ethical regulations}

Data were retrieved from the Netherlands Cancer Registry and Netherlands Pathology Registry (PALGA, the nationwide registry of pathology in the Netherlands [14]). The study was performed according to the cancer registry and pathology registry guidelines and national privacy regulations and approved by the medical ethical committee of the Maastricht University Medical Center (METC azM/UM 14-4-043, November 20, 2014).

\section{Patient selection}

All patients with a diagnosis of stage IV LCNEC recorded in either the cancer registry or the pathology registry between January 1, 2003 and December 31, 2012 were included. To select LCNEC from the cancer registry the International Classifications of Disease - Oncology 3rd edition code M8013 was used. Previously we have observed that a wide range of diagnostic terms are used to describe LCNEC [15]. To identify additional LCNEC cases in the cancer registry that had been diagnosed with alternative nomenclature, the additional diagnostic codes M8246 (neuroendocrine carcinoma) and M8574 (NSCLC with neuroendocrine differentiation) were included. Digital summaries of pathology reports retrieved from the pathology registry were screened for the diagnosis of LCNEC, as previously reported [15]. Patients diagnosed with metastatic LCNEC, including patients with tumours diagnosed with a nomenclature possibly referring to LCNEC, treated by chemotherapy retrieved from either of the national databases, were included. Data on the type of chemotherapy treatment was retrospectively updated in 2015 by qualified cancer registry data managers. Patients were excluded if details on chemotherapy were unavailable.

First, we analysed the type of chemotherapy in the selected patient study group (aim 1). We then performed a pathology review for all patients. Patients with a diagnosis based on cytology and patients for whom the original histopathological slides could not be retrieved were excluded. Overall survival and PFS were determined in patients with a panel-confirmed diagnosis of LCNEC (aim 2).

Support statement: This work was supported by a grant from the Dutch Cancer Society (number 7110). Funding information for this article has been deposited with the Crossref Funder Registry.

Conflict of interest: None declared. 
Data collection

Collected data included stage (tumour, node and metastasis (TNM) stage 6 or 7) and time from diagnosis to death or last follow-up censored for 36 months of overall survival. PFS was calculated from date of diagnosis until first evidence of progression, death or last day of follow-up. Treatment data included chemotherapy subtype, number of chemotherapy cycles and second-line treatment. First-line chemotherapy was clustered into three groups, as follows. 1) "NSCLC chemotherapy type" (NSCLC-t), consisting of gemcitabine, docetaxel, paclitaxel or vinorelbine; 2) "pemetrexed NSCLC type" (NSCLC-pt), with pemetrexed treatment only; and 3) "SCLC type" (SCLC-t), consisting of etoposide chemotherapy. The platinum components were either cisplatin or carboplatin. Metastatic sites at diagnosis were retrieved from documented clinical data (cTNM). Pathology data included pathology history, pathological specimen type and diagnosis according to the digital pathology report summary.

\section{Pathology revision}

Tumour histology slides were collected and included at least one immunohistochemical (IHC) neuroendocrine stain (CD56/NCAM, chromogranin-A or synaptophysin) and a haematoxylin and eosin stained slide. Review was performed by three pathologists (E. Thunnissen, R. van Sulyen and M. den Bakker), who were blinded for clinical outcome and original diagnosis. IHC staining patterns for neuroendocrine markers, cytokeratins, TTF1 and p63 and Ki-67 (if available) were assessed by J. Derks and R. van Sulyen prior to the central review meetings. The assessors evaluated haematoxylin and eosin slides at the multihead microscope; information on IHC expression patterns was provided (J. Derks). LCNEC was established when at least two pathologists agreed on the diagnosis, referred to as panel-consensus LCNEC. World Health Organization (WHO) 2015 criteria were evaluated for all panel-consensus established diagnoses. Additional detailed pathology review information can be found in the online supplementary pathology data file.

\section{Statistical analysis}

The Chi-squared and Fisher exact tests were used to compare categorical data. Continuous variables were tested using the Mann-Whitney U-test and the median and interquartile range (IQR) reported. Overall survival and PFS censoring took place at the closing date (February 1, 2014). Overall survival was estimated according to the Kaplan-Meier method and tested using the log-rank test. Multivariate Cox regression analysis was performed including covariates significant at univariate analysis. Nonproportionality was visually assessed by $\log$ minus $\log$ plots. Two-sided p-values $<0.05$ were considered significant. Analyses were performed using SPSS (version 22 for Windows; Chicago, IL, USA).

\section{Results}

\section{Population-based changes in chemotherapy treatment over time}

Data from 1627 patients from the cancer registry and 1172 patients from the pathology registry were retrieved. 355 patients had stage IV disease treated with chemotherapy. After excluding patients for whom details of chemotherapy treatment could not be retrieved, chemotherapy treatment was analysed in 294 patients (figure 1). A complete overview of retrieved diagnoses and chemotherapy treatment is presented in online supplementary table S1. NSCLC-t chemotherapy treatment in LCNEC significantly decreased over time from 59\% (2003-2009) to 31\% (2010-2012) ( $p<0.001)$; NSCLC-pt chemotherapy type increased from $10 \%$ to $16 \%(\mathrm{p}=0.29)$; and the SCLC type increased from $31 \%$ to $53 \%(\mathrm{p}=0.002)$.

\section{Panel-consensus diagnosed LCNEC}

Histopathological slides were retrieved from 207 patients. In 128 patients LCNEC was diagnosed by consensus, with 108 cases meeting all required WHO 2015 criteria (online supplementary table S2) [16]. Patients with a panel-confirmed LCNEC diagnosis $(\mathrm{n}=128)$ had a median age of 65 (56-71) years, 59\% were male and $67 \%$ were diagnosed by a (core) needle biopsy specimen (table 1). Metastases in the liver (53\%), bone $(27 \%)$ and nonmediastinal lymph nodes (22\%) were most common. Metastases confined to a single organ were present in $48 \%$ of patients. A minimum of four chemotherapy cycles (median (IQR) $4(2-4)$ ) were administered in $62 \%$ of patients. Second-line chemotherapy was administered in $23 \%$ of patients. Patients with more than three metastases in different organs more frequently received SCLC- $t$ chemotherapy. Overall, NSCLC-t chemotherapy was administered in $46 \%$ of patients, mainly platinumgemcitabine (76\% of NSCLC-t patients). NSCLC-pt and SCLC-t chemotherapy was administered in $16 \%$ and in $38 \%$ of patients, respectively. Characteristics of panel-consensus diagnosed LCNEC patients who fulfilled all required WHO criteria were not different and are described in online supplementary table S3.

\section{Overall survival in panel-consensus diagnosed LCNEC by chemotherapy cluster}

All but three patients died during the follow-up period. The median (95\% CI) overall survival was 7.3 months (6.3-8.2 months). Patients treated with NSCLC-t chemotherapy had a median overall survival of 8.5 months (7.0-9.9 months), which was significantly longer than for patients treated with NSCLC-pt 


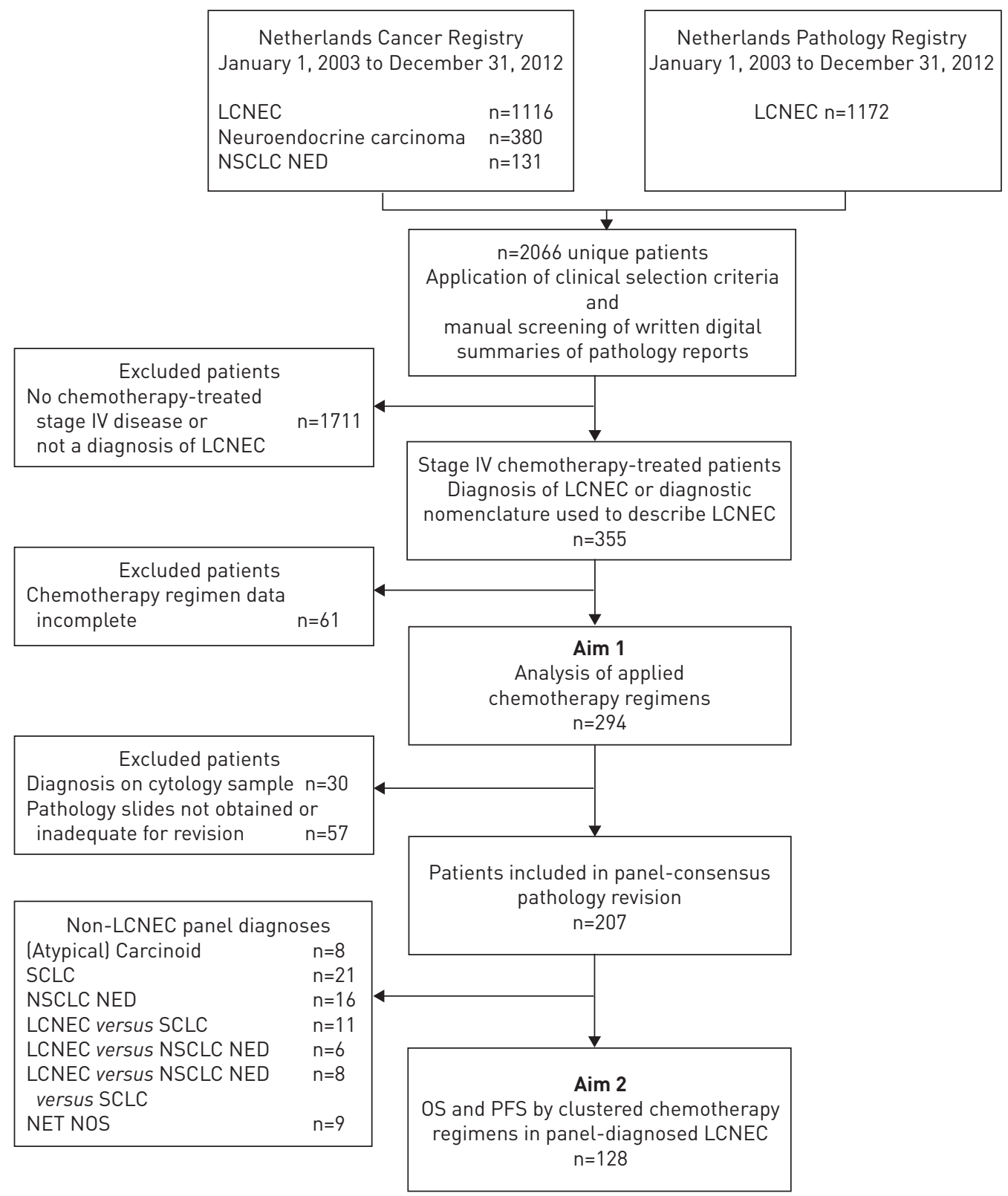

FIGURE 1 CONSORT (Consolidated Standards of Reporting Trials) diagram showing inclusion of patients and the performed pathology review. LCNEC: large cell neuroendocrine carcinoma; NSCLC NED: nonsmall cell lung carcinoma with immunohistochemically neuroendocrine differentiation; SCLC: small cell lung carcinoma; NET NOS: neuroendocrine tumour not otherwise specified; OS: overall survival; PFS: progression-free survival.

chemotherapy (5.9 months, 5.0-6.9 months; $\mathrm{p}=0.011$ ), and significantly longer than patients treated with SCLC-t chemotherapy (6.7 months, 5.0-8.5 months; $\mathrm{p}=0.012$ ) (figure 2a). In multivariate analysis, including the covariates significant at univariate analyses (sex, age, liver metastasis and number of organs with metastases at diagnosis) (online supplementary figure S2), results remained significant for NSCLC-t versus NSCLC-pt treatment (hazard ratio (HR) 2.51, 95\% CI 1.39-4.52; $\mathrm{p}=0.002$ ), and for NSCLC-t versus SCLC-t treatment $(1.66,1.08-2.56 ; \mathrm{p}=0.020)$ (figure 3). Cisplatinum versus carboplatinum compounds did not have a significant effect on the treatment outcome data (online supplementary figure S3). Corresponding results for overall survival and PFS in 108 patients with LCNEC in whose tumour samples all WHO 2015 criteria were confirmed are described in online supplementary figures S3, S4 and S5.

Overall survival in panel-consensus LCNEC according to chemotherapy subtype

Patients treated with platinum-gemcitabine chemotherapy had a median overall survival (95\% CI) of 7.8 months (5.9-9.6 months), which was significantly longer than for platinum-pemetrexed (5.9 months, 
TABLE 1 Clinical characteristics of patients with panel-consensus diagnosed large cell neuroendocrine carcinoma

\begin{tabular}{|c|c|c|c|c|c|c|}
\hline & Total cohort & \multicolumn{3}{|c|}{ Chemotherapy clusters } & \multicolumn{2}{|c|}{ p-value } \\
\hline Age years & $65(56-71)$ & $64(56-69)$ & $70(57-74)$ & $63(55-70)$ & $0.24^{\#}$ & $0.88^{\#}$ \\
\hline Male & 75 (59) & $33(55)$ & $12(60)$ & $30(63)$ & 0.70 & 0.43 \\
\hline Number of organs with metastases & & & & & $0.67^{1,+}$ & $0.012^{\uparrow,+}$ \\
\hline 3 & $11(9)$ & $2(3)$ & $0(0)$ & $9(19)$ & & \\
\hline$>3$ & $12(9)$ & $5(8)$ & $1(5)$ & $6(13)$ & & \\
\hline \multicolumn{7}{|l|}{ Organ metastases at diagnosis } \\
\hline Bone & $34(27)$ & $14(23)$ & $5(25)$ & $15(31)$ & 0.88 & 0.34 \\
\hline Liver & $68(53)$ & $30(50)$ & $10(50)$ & $28(58)$ & 1.00 & 0.39 \\
\hline Brain & $17(13)$ & $7(12)$ & $2(10)$ & $8(17)$ & 0.57 & 0.81 \\
\hline \multicolumn{7}{|l|}{ Nonclustered subtype of chemotherapy } \\
\hline Gemcitabine & $46(36)$ & $46(76)$ & & & & \\
\hline Paclitaxel & $7(5)$ & $7(12)$ & & & & \\
\hline Docetaxel & $6(5)$ & $6(10)$ & & & & \\
\hline Vinorelbine & $1(1)$ & $1(2)$ & & & & \\
\hline Etoposide & $48(38)$ & & & $48(100)$ & & \\
\hline Pemetrexed & $20(16)$ & & $20(100)$ & & & \\
\hline Cycles of chemotherapy & & & & & $0.30^{\S}$ & $0.09^{\S}$ \\
\hline 1 & $18(14)$ & $6(10)$ & $2(10)$ & $10(21)$ & & \\
\hline 2 & $15(12)$ & $5(8)$ & $4(20)$ & $6(13)$ & & \\
\hline 3 & $14(11)$ & $6(10)$ & $3(15)$ & $5(10)$ & & \\
\hline 4 & $63(49)$ & $30(50)$ & $11(55)$ & $22(46)$ & & \\
\hline
\end{tabular}

Data are presented as $\mathrm{n}(\%)$ or median (interquartile range), unless otherwise stated. Bold type represents statistical significance. NSCLC: nonsmall cell lung carcinoma; NSCLC-t: NSCLC cluster of gemcitabine, paclitaxel, docetaxel and vinorelbine chemotherapy; NSCLC-pt: NSCLC cluster of pemetrexed chemotherapy; SCLC-t: small cell lung carcinoma cluster of etoposide chemotherapy. "\#: Mann-Whitney U-test; ${ }^{\text {?: }}$ compared $\leqslant 2$ organ metastases with $>2$ organ metastases; ${ }^{+}$: Fisher exact test; ${ }^{\S}$ : compared $\leqslant 2$ cycles versus $\geqslant 3$ cycles of chemotherapy, excluding unknown cases.

5.0-6.9 months; $\mathrm{p}=0.019$ ) and for platinum-etoposide chemotherapy (6.7 months, 5.0-8.5 months; $\mathrm{p}=0.035$ ) (figure 2b). In multivariate analyses overall survival for gemcitabine was superior to pemetrexed chemotherapy (HR 2.39, 95\% CI 1.31-4.35; $\mathrm{p}=0.004$ ) and a strong trend was observed compared to etoposide (1.54, 0.97-2.43; $\mathrm{p}=0.066$ ) (figure 3). Paclitaxel-treated patients had a median overall survival of 8.7 months (95\% CI 2.7-14.7 months), significantly longer than for pemetrexed chemotherapy $(p=0.034)$, and a strong trend was observed for etoposide chemotherapy $(\mathrm{p}=0.057)$ (figure $2 b)$. In multivariate analysis paclitaxel showed superior overall survival compared to pemetrexed chemotherapy (HR 4.04, 95\% CI 1.46-11.22; $\mathrm{p}=0.007$ ) and etoposide chemotherapy treatment (HR 2.60, 95\% CI 1.07-6.35; $\mathrm{p}=0.035$ ) (figure 3 ).

PFS in panel-consensus LCNEC according to chemotherapy subtype

Data on PFS were available in 119 patients; all except one patient progressed or died during the study period. The median PFS (95\% CI) was 4.7 months (4.2-5.3 months). Only NSCLC-pt chemotherapy treated patients had a significantly worse PFS (4.1 months, 3.8-4.5 months; $\mathrm{p}=0.040)$ compared to patients treated with NSCLC-pt chemotherapy (figure 4a). Patients treated with gemcitabine chemotherapy had a significantly longer PFS of 5.2 months (4.1-6.2 months) compared to patients treated with NSCLC-pt chemotherapy $(\mathrm{p}=0.034)$ (figure $4 \mathrm{~b})$. All other comparisons of specific subtypes of chemotherapy showed no significant differences in PFS. 

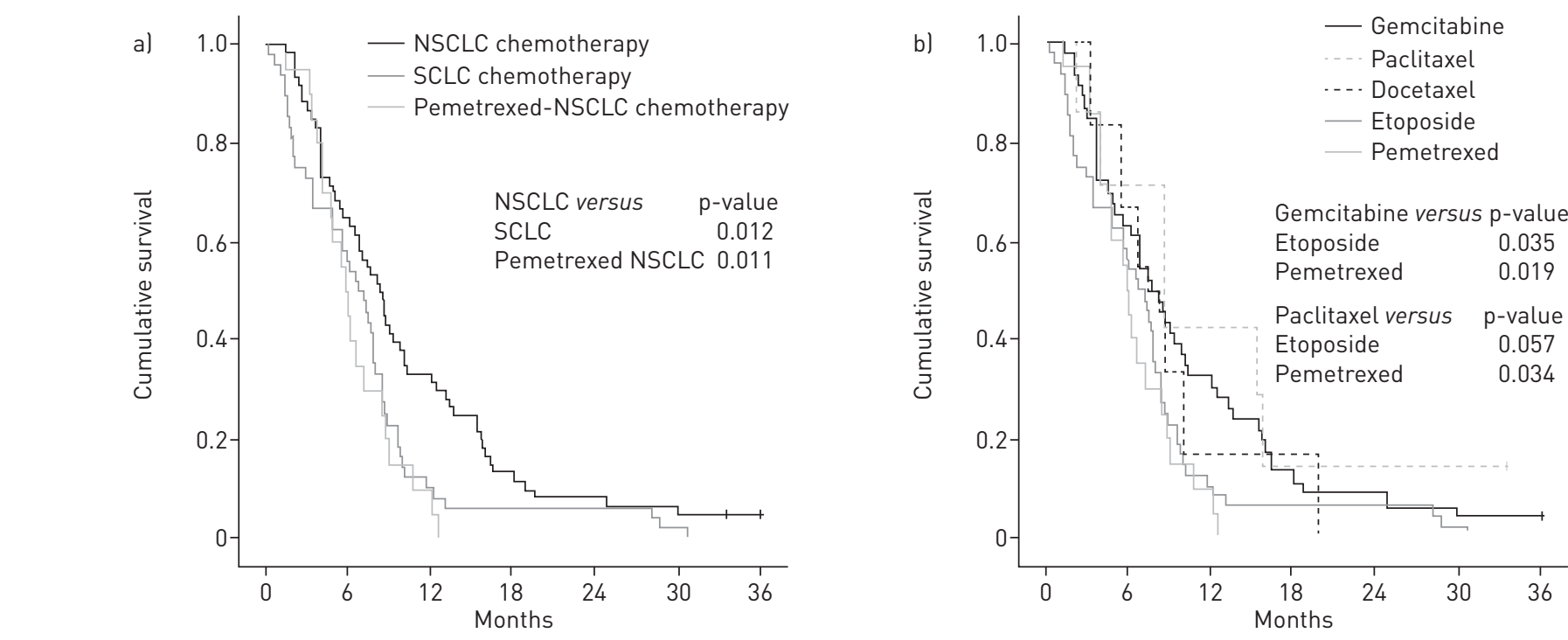

$\begin{array}{lllcllll}\text { Patients at risk n } & & & & & & & \\ \text { NSCLC } & 60 & 39 & 20 & 8 & 5 & 3 & 2 \\ \text { SCLC } & 48 & 27 & 5 & 3 & 3 & 1 & 0 \\ \text { Pemetrexed-NSCLC } & 20 & 10 & 2 & 0 & 0 & 0 & 0\end{array}$

$\begin{array}{lccccccc}\text { Patients at risk } \mathrm{n} & & & & & & & \\ \text { Gemcitabine } & 46 & 29 & 15 & 6 & 4 & 2 & 2 \\ \text { Paclitaxel } & 7 & 5 & 3 & 1 & 1 & 1 & 0 \\ \text { Docetaxel } & 6 & 4 & 1 & 1 & 0 & 0 & 0 \\ \text { Etoposide } & 48 & 27 & 5 & 3 & 3 & 1 & 0 \\ \text { Pemetrexed } & 20 & 10 & 2 & 0 & 0 & 0 & 0\end{array}$

FIGURE 2 Overall survival in panel-consensus diagnosed large cell neuroendocrine carcinoma patients compared for a) chemotherapy clusters and b) subtypes of chemotherapy (excluding vinorelbine). $n=128$. NSCLC: nonsmall cell lung carcinoma regimen; SCLC: small cell lung carcinoma regimen.

\section{Discussion}

Patients treated with doublet combined chemotherapy for metastatic LCNEC have a poor survival and the optimal chemotherapy treatment for LCNEC remains unascertained. Here we report that patients treated with NSCLC-t chemotherapy, mainly gemcitabine, have superior overall survival compared with patients treated with NSCLC-pt chemotherapy. In addition, the combination of NSCLC-t regimens, excluding those containing pemetrexed, showed superior survival compared with etoposide (SCLC-t) chemotherapy. These results contrast with the advised treatment in the ASCO guideline [7].

Chemotherapy treatment for patients with LCNEC changed significantly between 2003 and 2012 in the Netherlands, with a decrease in NSCLC-t chemotherapy and an increase in SCLC-t chemotherapy. This observation corresponds with data accrued from a 2014 questionnaire survey circulated among 21 Dutch pulmonary physicians. In this survey the majority of physicians (80\%) would treat LCNEC with SCLC chemotherapy (online supplementary figure S6). We were unable to find specific explanations why the treatment of LCNEC has changed. Treatment preferences may have been influenced by a study published in 2005 describing the favourable response of LCNEC to SCLC-type chemotherapy [17].

Several studies have evaluated chemotherapy in LCNEC, but the reported studies are heterogeneous in case selection and confirmation of the pathology diagnosis (table 2). Two phase II trials, both with pathology review, have been reported. A European trial [11] reported a median overall survival of 8.0 months (95\% CI 3.7-7.9 months), a PFS of 5.0 months (95\% CI 4.0-7.9 months) and an ORR of 34\% in 29 patients treated with platinum-etoposide chemotherapy. In a Japanese trial [12], a median overall survival of 12.6 (95\% CI 9.3-16.0) months, PFS of 5.8 (95\% CI 3.8-7.8) months and an ORR of 47\% was reported for treatment with platinum-irinotecan $(n=30)$. In retrospectively evaluated cohorts of LCNEC patients, the reported ORR for platinum-etoposide chemotherapy ranged from $37 \%$ to $73 \%$ and overall survival ranged from 8.4 to 16.5 months [17-20]. Treatment outcomes for SCLC- and NSCLC-type chemotherapy for LCNEC has previously been evaluated; 27 patients showed an improved survival for platinum-etoposide chemotherapy compared to a combination of NSCLC regimens [17]. Conversely, evaluation of an additional 26 patients showed a significantly lower overall survival for platinum-etoposide chemotherapy compared to a combination of NSCLC regimens [19]. Because NSCLC regimens are frequently combined for analysis, there is a lack of data on subtype-specific overall survival and PFS. The reported ORRs for platinum combined with gemcitabine, docetaxel and paclitaxel are $41 \%(n=17), 77 \%(n=9)$ and $81 \%(n=11)$, respectively $[18,21]$.

Platinum-pemetrexed chemotherapy is advised as first-line treatment in patients with metastatic nonsquamous NSCLC [7]. However, platinum-pemetrexed chemotherapy showed inferior results compared 


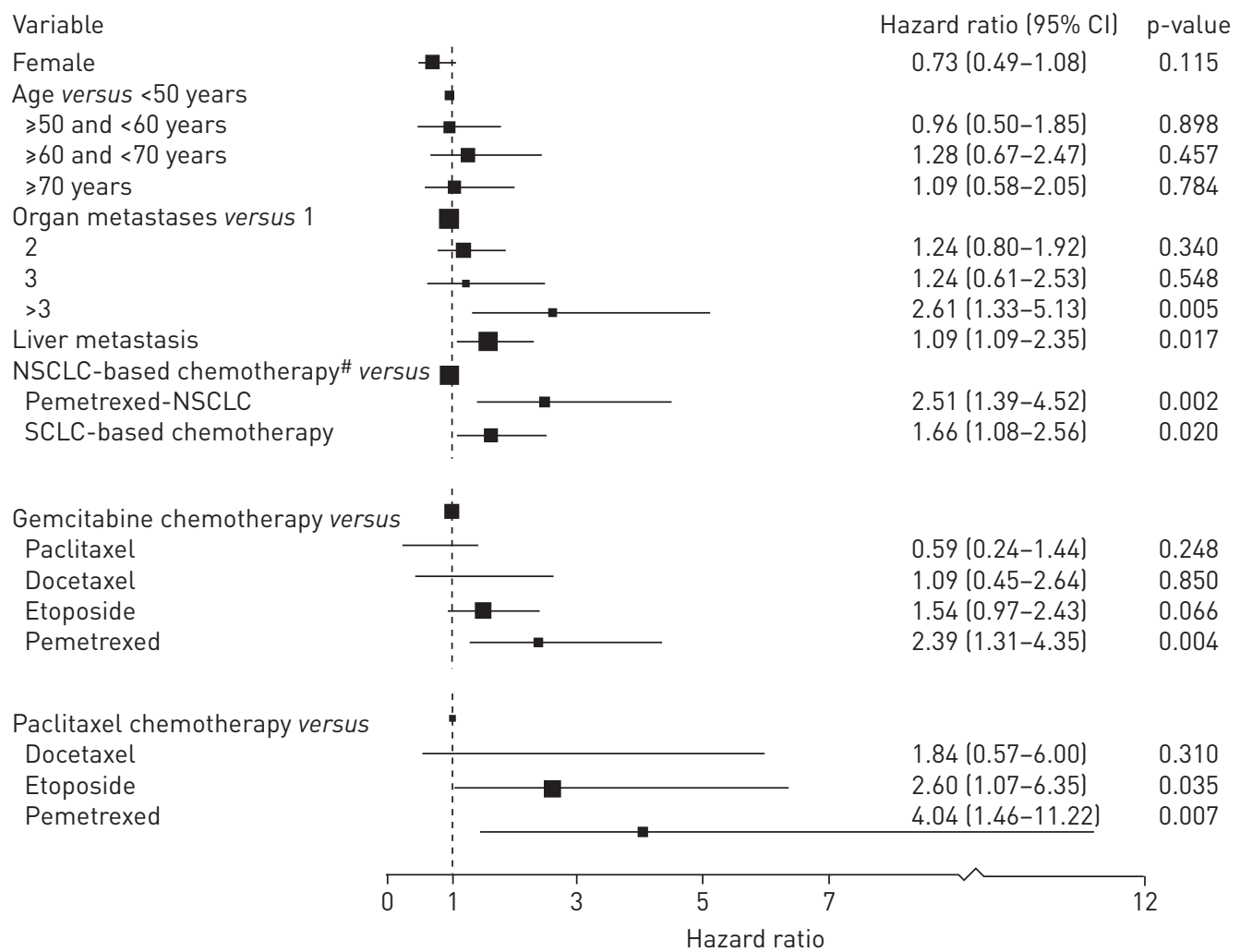

FIGURE 3 Three multivariate models are presented for clustered chemotherapy, platinum-gemcitabine and platinum-paclitaxel chemotherapy in panel-consensus large cell neuroendocrine carcinoma. $\mathrm{n}=128$. NSCLC: nonsmall cell lung carcinoma; SCLC: small cell lung carcinoma. " : excluding vinorelbine.

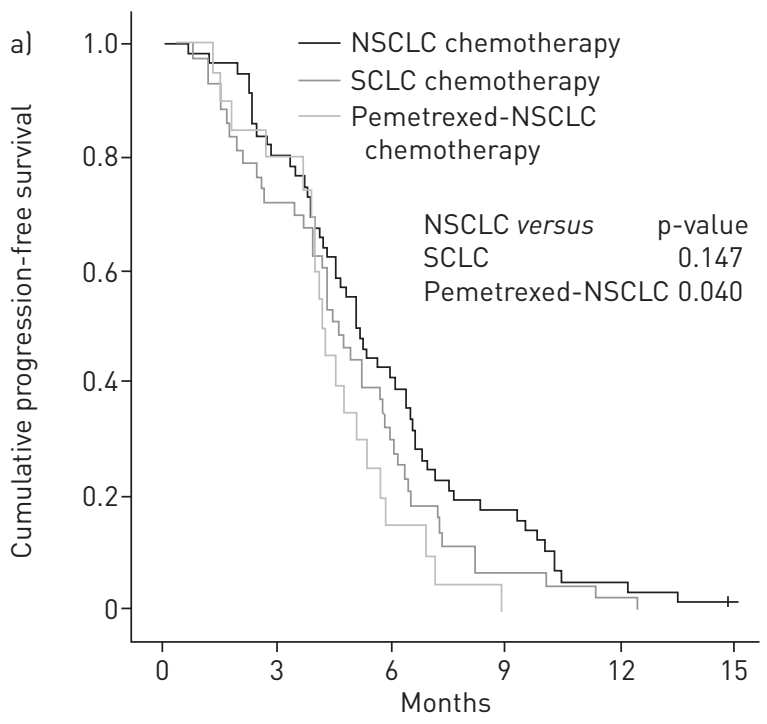

Patients at risk $n$

$\begin{array}{lllllll}\text { NSCLC } & 56 & 48 & 24 & 11 & 4 & 2 \\ \text { SCLC } & 43 & 31 & 13 & 3 & 1 & 0 \\ \text { Pemetrexed-NSCLC } & 20 & 16 & 3 & 0 & 0 & 0\end{array}$

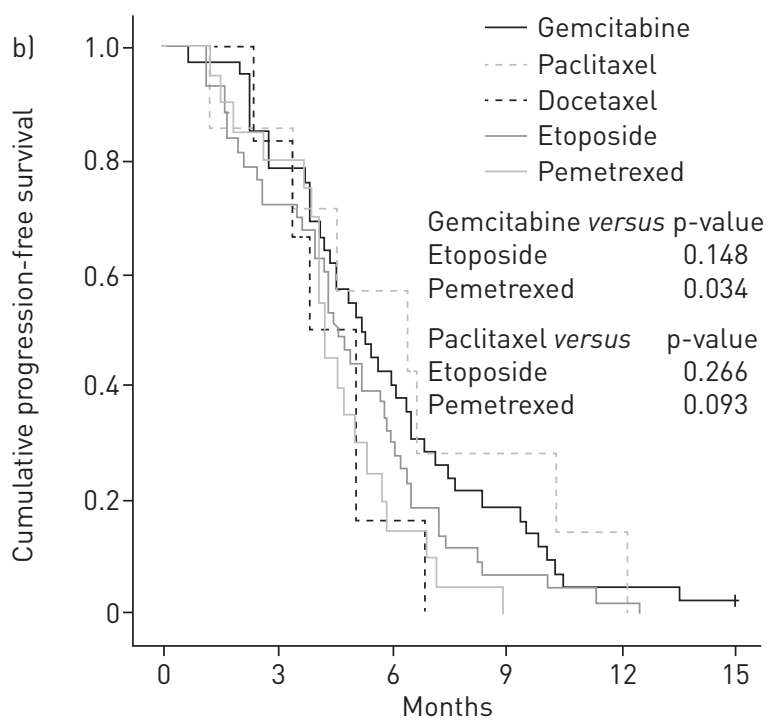

Patients at risk $\mathrm{n}$

$\begin{array}{lcccccc}\text { Gemcitabine } & 42 & 36 & 18 & 9 & 3 & 2 \\ \text { Paclitaxel } & 7 & 6 & 4 & 2 & 1 & 0 \\ \text { Docetaxel } & 6 & 5 & 1 & 0 & 0 & 0 \\ \text { Etoposide } & 43 & 31 & 13 & 3 & 1 & 0 \\ \text { Pemetrexed } & 20 & 16 & 3 & 0 & 0 & 0\end{array}$

FIGURE 4 Progression-free survival compared for a) chemotherapy clusters and b) subtypes of chemotherapy (excluding vinorelbinel in panel-consensus large cell neuroendocrine carcinoma. $n=119$. NSCLC: nonsmall cell lung carcinoma chemotherapy regimen; SCLC: small cell lung carcinoma chemotherapy regimen. 
TABLE 2 Overview of response to chemotherapy in advanced-stage large cell neuroendocrine carcinoma disease

\begin{tabular}{|c|c|c|c|c|c|c|c|c|c|}
\hline First author [reference] & Design & $\begin{array}{l}\text { Panel review } \\
\text { (pathologists } n \text { ) }\end{array}$ & $\begin{array}{l}\text { Inclusion } \\
\text { period }\end{array}$ & \multicolumn{3}{|c|}{ NSCLC chemotherapy } & \multicolumn{3}{|c|}{ SCLC chemotherapy } \\
\hline Le Treut [11] & $P$ & Yes (?) & $2004-2009$ & & & & 29 & 34 & 8.0 \\
\hline Nıно [12] & $P$ & Yes (6) & $2005-2011$ & & & & 30 & 47 & 12.6 \\
\hline Metro [20] & $\mathrm{R}$ & No revision & Unclear & & & & 37 & 43 & 8.4 \\
\hline Naldoo [19] & $\mathrm{R}$ & Yes (3) & $2006-2013$ & $11^{\#}$ & $0^{\#}$ & 19.5 & $26^{\pi}$ & 37 & 8.3 \\
\hline FuJIWARA [21] & $\mathrm{R}$ & No (1) & $1999-2006$ & $9^{\S}$ & 77 & & 13 & 46 & \\
\hline DERKs (present study) & $\mathrm{R}$ & Yes (3) & 2003-2012 & $\begin{array}{c}60 \text { (NSCLC) } \\
20 \text { (pemetrexed- } \\
\text { NSCLC) }\end{array}$ & & $\begin{array}{l}8.5 \\
5.9\end{array}$ & 48 & 33 & 6.7 \\
\hline
\end{tabular}

Studies including patients treated with chemoradiotherapy are not shown. NSCLC: nonsmall cell lung carcinoma; SCLC: small cell lung carcinoma; ORR: objective response rate; OS: overall survival; P: prospective; R: retrospective. \#: four patients were evaluated according to response evaluation criteria in solid tumours (RECIST), including temozolomide $(n=2)$, pemetrexed $(n=1)$ and platinum combined with everolimus $(n=1)$; ${ }^{~}: 19$ patients were evaluated according to RECIST criteria; ${ }^{+}$: including gemcitabine-platinum ( $\left.n=17\right)$, taxane-platinum ( $\left.n=4\right)$, tyrosine kinase inhibitor $(n=2)$ and other platinum $(n=11) ;{ }^{\S}$ : taxane combined with platinum $(n=7)$, taxane monotherapy $(n=1)$ and platinum-vinorelbine $(n=1)$.

to platinum-etoposide in SCLC [22], a tumour biologically closely related to LCNEC. The poor therapeutic response of pemetrexed may be due to the reported high expression of the thymidylate synthesis (TS) gene in LCNEC. Increased TS expression is suggested to be related to resistance to pemetrexed therapy [23-25]. The increased tendency for pemetrexed resistance coupled with the reported clinical observations suggest that pemetrexed should not be used in patients with LCNEC.

Molecular changes in LCNEC and SCLC have been described. SCLC is characterised by RB1 and TP53 gene mutations, whereas LCNEC was characterised by mutually exclusive RB1 and TP53 gene inactivation versus a combination of STK11/KRAS/KEAP1 gene mutations $[9,26]$. In future studies it would be of interest to analyse these patterns to investigate whether the molecular background corresponds with responses to different chemotherapy regimens [9].

This study has several limitations. First, it is a retrospective study and chemotherapy data could not be retrieved in all patients. However, the exclusion of patients was random and not by selection, as evidenced by the similar overall survival and age range of excluded patients compared to the analysed patient cohort (online supplementary tables S4 and S5). Second, information on WHO performance score was lacking, and this may have confounded reported overall survival. We observed no differences in overall survival for treatment with cisplatinum or carboplatinum chemotherapy (online supplementary figure S2). Third, completion of chemotherapy cycles differed slightly between the NSCLC-t and SCLC-t treatments. Nevertheless, up to $62 \%$ of patients completed four or more cycles of chemotherapy and this was not significantly different between treatment groups. Fourth, the reported overall survival for chemotherapy-treated subtypes may have been confounded by strong therapeutic effects of second-line treatment. However, in the presented cohort the frequency of second-line treatment was relatively low (23\%) and not statistically different among clustered chemotherapy subtypes (table 1). The frequency of second-line treatment is lower than reported in a Japanese phase II trial (86\%) [12], but not much lower than reported for daily clinical practice in lung cancer (32\%) [27]. Finally, data on PFS were obtained retrospectively and could not be formally evaluated by the RECIST (response evaluation criteria in solid tumours) criteria, as this was analysed in a real-world setting and not in a clinical trial. Response evaluation was not standardised and incomplete in $40 \%$ of patients; therefore, these data are not reported.

In conclusion, we present the largest series of patients with pathology-reviewed metastatic LCNEC to date, and show that NSCLC-t regimens, mainly platinum-gemcitabine chemotherapy, are superior to platinumpemetrexed and platinum-etoposide treatment. These results need prospective evaluation, ideally in a randomised trial, in centrally confirmed LCNEC.

\section{Acknowledgements}

The members of the PALGA (the nationwide network and registry of histo- and cytopathology in the Netherlands) group are as follows. L. Arensman: Meander Medisch Centrum Klinische Pathologie (Amersfoort); F.E. Bellot: Klinische Pathologie Hoofddorp (Hoofddorp); J.E. Broers: Isala Klinieken (Zwolle); C.M. van Dish: Klinische Pathologie Groene Hart Ziekenhuis 
(Gouda); K.E.S. Duthoi: Amphia Ziekenhuis (Breda); M.J. Flens: Symbiant (Zaandam); J.M.M. Grefte: Gelre Ziekenhuis Klinische Pathologie (Apeldoorn); M.C.H. Hogenes: Labpon (Hengelo); R. Natté: Haga Ziekenhuis (den Haag); A.F. van Hamel: Pathologie SSZOG (Groningen); P.J.J.M. Klinkhamer: Stichting PAMM (Eindhoven); J.W.R. Meijer: Ziekenhijs Rijnstate (Arnhem); J.C.C. van der Meij: Pathologie Friesland (Leeuwarden); F.H. van Nederveen: Laboratorium voor Pathologie (PAL) (Dordrecht); E.W.P. Nijhuis: Onze Lieve Vrouwe Gasthuis (Amsterdam); M.F.M. van Oosterhout: St. Antonius Ziekenhuis (Nieuwegein); S.H. Sastrowijoto: Pathologie Sittard (Sittard); K. Schelfout: Stichting Pathologisch en Cytologisch Laboratorium West-Brabant (Bergen-op-Zoom); J. Sietsma: Pathologie Martini Ziekenhuis (Groningen); F.M.M. Smedts: Reinier Haga MDC (Delft); M.M. Smits: Laboratorium voor Klinische Pathologie (Deventer); J. Stavast: Laboratorium Klinische Pathologie Centraal Brabant (Tilburg); W. Timens: Pathologie UMCG (Groningen); M.L. van Velthuysen: NKI-AVL (Amsterdam); A. Vink: Universitair Medisch Centrum Utrecht (Utrecht); C.C.A.P. Wauters: Canisius Wilhelmina Ziekenhuis (Nijmegen); S. Wouda: VieCuri Medical Centre (Venlo).

This work has been presented at European Society for Medical Oncology poster discussion, Copenhagen, Denmark, October 7-11, 2016 (abstract 1122) and European Neuroendocrine Tumor Society Conference, Barcelona, Spain, March 8-10, 2017.

\section{References}

1 Travis WD, Linnoila RI, Tsokos MG, et al. Neuroendocrine tumors of the lung with proposed criteria for large-cell neuroendocrine carcinoma. An ultrastructural, immunohistochemical, and flow cytometric study of 35 cases. Am J Surg Pathol 1991; 15: 529-553.

2 Derks JL, Hendriks LE, Buikhuisen WA, et al. Clinical features of large cell neuroendocrine carcinoma: a population-based overview. Eur Respir J 2016; 47: 615-624.

3 Den Bakker MA, Willemsen S, Grünberg K, et al. Small cell carcinoma of the lung and large cell neuroendocrine carcinoma interobserver variability. Histopathology 2010; 56: 356-363.

4 Thunnissen E, Borczuk AC, Flieder DB, et al. The use of immunohistochemistry improves the diagnosis of small cell lung cancer and its differential diagnosis. An international reproducibility study in a demanding set of cases. J Thorac Oncol 2017; 12: 334-346.

5 Fasano M, Della Corte CM, Papaccio F, et al. Pulmonary large-cell neuroendocrine carcinoma: from epidemiology to therapy. J Thorac Oncol 2015; 10: 1133-1141.

6 Reck M, Popat S, Reinmuth N, et al. Metastatic non-small-cell lung cancer (NSCLC): ESMO clinical practice guidelines for diagnosis, treatment and follow-up. Ann Oncol 2014; 25: Suppl. 3, iii27-iii39.

7 Masters GA, Temin S, Azzoli CG, et al. Systemic therapy for stage IV non-small-cell lung cancer: American Society of Clinical Oncology clinical practice guideline update. J Clin Oncol 2015; 33: 3488-3515.

8 George J, Lim JS, Jang SJ, et al. Comprehensive genomic profiles of small cell lung cancer. Nature 2015; 524: 47-53.

9 Rekhtman N, Pietanza MC, Hellmann M, et al. Next-generation sequencing of pulmonary large cell neuroendocrine carcinoma reveals small cell carcinoma-like and non-small cell carcinoma-like subsets. Clin Cancer Res 2016; 22: 3618-3629.

10 Asamura H, Kameya T, Matsuno Y, et al. Neuroendocrine neoplasms of the lung: a prognostic spectrum. J Clin Oncol 2006; 24: 70-76.

11 Le Treut J, Sault MC, Lena H, et al. Multicentre phase II study of cisplatin-etoposide chemotherapy for advanced large-cell neuroendocrine lung carcinoma: the GFPC 0302 study. Ann Oncol 2013; 24: 1548-1552.

12 Niho S, Kenmotsu H, Sekine I, et al. Combination chemotherapy with irinotecan and cisplatin for large-cell neuroendocrine carcinoma of the lung: a multicenter phase II study. J Thorac Oncol 2013; 8: 980-984.

13 Rossi A, Di Maio M, Chiodini P, et al. Carboplatin- or cisplatin-based chemotherapy in first-line treatment of small-cell lung cancer: the COCIS meta-analysis of individual patient data. J Clin Oncol 2012; 30: 1692-1698.

14 Casparie M, Tiebosch AT, Burger G, et al. Pathology databanking and biobanking in the Netherlands, a central role for PALGA, the nationwide histopathology and cytopathology data network and archive. Cell Oncol 2007; 29: 19-24.

15 Derks JL, van Suylen RJ, Thunnissen E, et al. A population-based analysis of application of WHO nomenclature in pathology reports of pulmonary neuroendocrine tumors. J Thorac Oncol 2016; 11: 593-602.

16 Travis WD, Brambilla E, Burke AP, et al. WHO Classification of Tumours of the Lung, Pleura, Thymus and Heart. 4th Edn. Lyon, International Agency for Research on Cancer, 2015.

17 Rossi G, Cavazza A, Marchioni A, et al. Role of chemotherapy and the receptor tyrosine kinases KIT, PDGFR $\alpha$, PDGFR $\beta$, and Met in large-cell neuroendocrine carcinoma of the lung. J Clin Oncol 2005; 23: 8774-8785.

18 Sun JM, Ahn MJ, Ahn JS, et al. Chemotherapy for pulmonary large cell neuroendocrine carcinoma: similar to that for small cell lung cancer or non-small cell lung cancer? Lung Cancer 2012; 77: 365-370.

19 Naidoo J, Santos-Zabala ML, Iyriboz T, et al. Large cell neuroendocrine carcinoma of the lung: clinico-pathologic features, treatment, and outcomes. Clin Lung Cancer 2016; 17: e121-e129.

20 Metro G, Ricciuti B, Chiari R, et al. Survival outcomes and incidence of brain recurrence in high-grade neuroendocrine carcinomas of the lung: implications for clinical practice. Lung Cancer 2016; 95: 82-87.

21 Fujiwara Y, Sekine I, Tsuta K, et al. Effect of platinum combined with irinotecan or paclitaxel against large cell neuroendocrine carcinoma of the lung. Jpn J Clin Oncol 2007; 37: 482-486.

22 Socinski MA, Smit EF, Lorigan P, et al. Phase III study of pemetrexed plus carboplatin compared with etoposide plus carboplatin in chemotherapy-naive patients with extensive-stage small-cell lung cancer. J Clin Oncol 2009; 27: 4787-4792.

23 Smit EF, Socinski MA, Mullaney BP, et al. Biomarker analysis in a phase III study of pemetrexed-carboplatin versus etoposide-carboplatin in chemonaive patients with extensive-stage small-cell lung cancer. Ann Oncol 2012; 23: 1723-1729.

24 Sun JM, Ahn JS, Jung SH, et al. Pemetrexed plus cisplatin versus gemcitabine plus cisplatin according to thymidylate synthase expression in nonsquamous non-small-cell lung cancer: a biomarker-stratified randomized phase II trial. J Clin Oncol 2015; 33: 2450-2456.

25 Monica V, Scagliotti GV, Ceppi P, et al. Differential thymidylate synthase expression in different variants of large-cell carcinoma of the lung. Clin Cancer Res 2009; 15: 7547-7552.

26 Fernandez-Cuesta L, Peifer M, Lu X, et al. Cross-entity mutation analysis of lung neuroendocrine tumors sheds light into their molecular origin and identifies new therapeutic targets. Cancer Res 2014; 74: abstract 1531.

27 Sacher AG, Le LW, Lau A, et al. Real-world chemotherapy treatment patterns in metastatic non-small cell lung cancer: are patients undertreated? Cancer 2015; 121: 2562-2569. 